Int. J. Dev. Biol. 62: 527-535 (2018)

https://doi.org/10.1387/ijdb.180066bp

\title{
Myths vs. FACS: what do we know about planarian stem cell lineages?
}

\author{
ALYSSA M. MOLINARO ${ }^{1,2}$ and BRET J. PEARSON*,1,2,3 \\ ${ }^{1}$ Hospital for Sick Children, Program in Developmental and Stem Cell Biology, ${ }^{2}$ Department of Molecular Genetics, \\ University of Toronto and ${ }^{3}$ Ontario Institute for Cancer Research, Toronto, ON, Canada
}

\begin{abstract}
Historically, planarian neoblasts were thought to be a homogeneous population of pluripotent stem cells; however, recent population and single-cell level analyses have refuted this idea. Evidence for lineage commitment at the neoblast level has been provided via a number of independent studies using a variety of methods. In situ hybridization experiments first demonstrated the co-expression of lineage-specific markers in neoblasts (marked by piwi-1 expression) isolated by FACS. Subsequently, single cell transcriptomic analyses of FACS-isolated neoblasts uncovered broad lineage-primed neoblast classes based on the clustering of transcriptional profiles and expression of known tissue-specific markers. Additionally, single neoblast pluripotency (and fate restriction) has been demonstrated by single cell transplantation experiments into neoblast-void animals. Here we look to recount the current status of the planarian neoblast field and offer discussion on the caveats of neoblast biology and how to address them experimentally.
\end{abstract}

KEY WORDS: stem cell, neoblast, planarian, clonogenic, lineage

\section{Introduction}

Humans replace billions of cells every day through the process of normal physiological cell turnover (homeostasis), and all of the new cells are ultimately the product of adult stem cells (ASCs) (Pellettieri and Alvarado, 2007). ASCs are central to several aspects of human biology such as tumor suppression, regeneration (or lack thereof), and aging, yet ASCs are extraordinarily difficult to study in the lab (with few exceptions, such as hematopoietic stem cells). In vitro, ASCs have highly asymmetric cell lineage kinetics, so they are difficult to expand in culture (Sherley, 2002). In vivo models either tend to have few ASCs or have ASCs that are very difficult to visualize or manipulate. For these reasons, ASCs remain understudied.

The freshwater planarian is a free-living member of the phylum Platyhelminthes in the Lophotrochozoan superphylum and has recently gained traction as an in vivo system to study ASC biology. Planarians are one of the best regenerators in nature. In just 7 days, a tiny planarian body fragment can fully regenerate and correctly pattern all missing tissues to create a functional, proportional worm (Cebrià, 2007; Newmark and Alvarado, 2002; Reddien and Alvarado, 2004). Impressively, the asexually reproducing strain of Schmidtea mediterranea has been propagated in laboratories for over a decade by repeated cycles of amputation and regeneration, highlighting the essentially immortal nature of these flatworms. This regenerative ability is supported by an abundant population of ASCs, called neoblasts, present throughout the planarian body (Dubois, 1949; Pellettieri and Alvarado, 2007; Randolph, 1892; Randolph, 1897; Rink, 2013; Zhu and Pearson, 2016). Neoblasts were historically considered a homogeneous population of pluripotent stem cells, but the dawn of single-cell transcriptomic analysis has brought with it evidence of heterogeneous, lineage-primed neoblast classes as well as a myriad of committed progenitor cells (Cowles et al., 2013; Currie et al., 2016; Currie and Pearson, 2013; Lapan and Reddien, 2012; Molinaro and Pearson, 2016; Scimone et al., 2014; van Wolfswinkel et al., 2014).

As the only cycling cells in planarians, neoblasts can be identified as cells with >2C DNA content (i.e. cells in the S/G2/M phases of the cell cycle), which are rapidly lost following lethal irradiation due to DNA damage (Eisenhoffer et al., 2008; Newmark and Alvarado, 2000; Reddien et al., 2005). These properties enable the prospective isolation of cycling neoblasts using fluorescence activated cell sorting (FACS). Developed by Hayashi et al., this technique relies on the irradiation sensitivity of neoblasts combined with cell

Abbreviations used in this paper: ASC, adult stem cell; FACS, fluorescence-activated cell sorting; FISH, fluorescent in situ hybridization; RNAi, RNA-interference; scRNAseq, single-cell RNA-sequencing.

\footnotetext{
*Address correspondence to: Bret J. Pearson. Peter Gilgan Centre for Research and Learning, 686 Bay Street, 18.9712, Toronto, ON M5G 0A4, Canada. Tel: 416-813-7654 ×328370. E-mail: bret.pearson@sickkids.ca - (iD) https://orcid.org/0000-0002-3473-901X
} 
cycle analysis using the DNA dye Hoechst (Hayashi et al., 2006). With this strategy, a population of Hoechst ${ }^{\text {hi }}$ cells is reproducibly lost within 24 hours post-irradiation (hpi) (Fig. 1 A,B). Referred to as the X1 FACS gate, these cells exhibit enriched expression of cell cycle markers and at least $90 \%$ express piwi-1; therefore, this gate is considered to be the "stem cell" gate (Eisenhoffer et al., 2008; Hayashi et al., 2006; Reddien et al., 2005). Due to the lack of transgenesis and absence of cell-surface antibodies in planarians, this Hoechst-based FACS isolation strategy remains the only method for prospective isolation of neoblasts. Thus, in the experiments leading to the major neoblast class discoveries discussed below, the definition of these cells as neoblasts was based initially on their isolation from the $\mathrm{X} 1 \mathrm{gate}$, with subsequent molecular verification using known neoblast markers such as the Argonaut-family homologs piwi-1 and piwi-2(Reddien et al., 2005).

In a landmark study from almost a decade ago, Eisenhoffer et al., demonstrated the enrichment of "progeny markers" in another irradiation-sensitive population of cells referred to as the "X2" FACS gate (Fig 1 A,B) (Eisenhoffer et al., 2008). The X2 gate resembles a classic Hoechst side population as these cells register as having $<2 \mathrm{C}$ DNA content, which is due to Hoechst efflux (Hayashi et al., 2006; Pearson and Alvarado, 2010). This can be demonstrated by flooding the cells with 5 -times the normal Hoechst concentration, which results in a shift of this gate toward 2C DNA content. This indicates that the low Hoechst fluorescence normally observed is due to efflux of the dye, which is commonly seen in classic side populations (Fig 1C). Following a lethal dose of irradiation, the actively cycling neoblasts of the $\mathrm{X} 1$ gate are quickly eliminated, followed closely by depletion of cells in the $\mathrm{X} 2$ gate and leaving behind the $\mathrm{X}$-insensitive (Xins) gate, which contains irradiation-insensitive, mature cell types from most tissues (Fig 1 A,B) (Eisenhoffer et al., 2008; Hayashi et al., 2006). From this observation, the authors reasoned that irradiation-depletion kinetics could serve as a proxy for cellular differentiation state, and thus the X2 gate is often considered the "progeny" gate. That said, how neoblasts progress through these FACS gates after cell division is not precisely known. Interestingly, the expression level of some Xins-enriched genes has been observed to decrease after irradiation, a phenomenon that does not entirely fit with its accepted description as the "mature cell" gate (Zhu et al., 2015).
It is also unclear whether $\mathrm{G} 1$ neoblasts reside in the $\mathrm{X} 2$ gate (in which piwi-1 expression is detectable, albeit at much lower levels than in the X1 gate (Labbé et al., 2012)) or in between the X1 and $X 2$ gates in an understudied region of the FACS plot that also contains irradiation-sensitive cells. To date, nearly all transcriptomic work on planarian neoblasts has focused on the $\mathrm{X} 1$ gate.

This review will summarize what is known about the neoblast classes and explore some interesting caveats of planarian neoblast biology. Are the lineage-primed neoblast classes bona fide stem cell populations? What exactly are the cell types in each FACS gate? If it is a distinct cell type, how do we find the pluripotent neoblast in the FACS cloud? What are the techniques and tools needed in the future to answer questions definitively about neoblast identity and cellular potential? Here we speculate about the answers to these questions and discuss how they might be addressed experimentally.

\section{Lineage-primed neoblast classes}

The original morphology-based characterization of the seemingly homogeneous neoblast population obscured a plethora of underlying molecular heterogeneity for decades, but recent advances in molecular techniques such as in situ hybridization (ISH) have been exceptionally revealing. The last 10 years have seen tremendous progress in the field of planarian neoblast biology, and thus far, there have been five major neoblast classes described in S. mediterranea: zeta neoblasts of the epithelial lineage, gamma neoblasts of the gut lineage, nu neoblasts of the neural lineage, the pluripotent sigma class, and finally, an experimentally verified clonogenic neoblast class (cNeoblasts) (Molinaro and Pearson, 2016; Wagner et al., 2011; van Wolfswinkel et al., 2014). With the exception of cNeoblasts, these neoblast classes were identified through single-cell transcriptomic analyses and some putative molecular markers have been described. The cNeoblast was identified through a series of elegant cell transplantation experiments, in which a small percentage of transplanted cells were able to clonally expand and rescue lethally-irradiated hosts (Wagner et al., 2011). Molecular markers for cNeoblasts have not yet been identified, and prospective isolation using FACS has not yet been achieved for any neoblast class. Thus, studying the roles
A

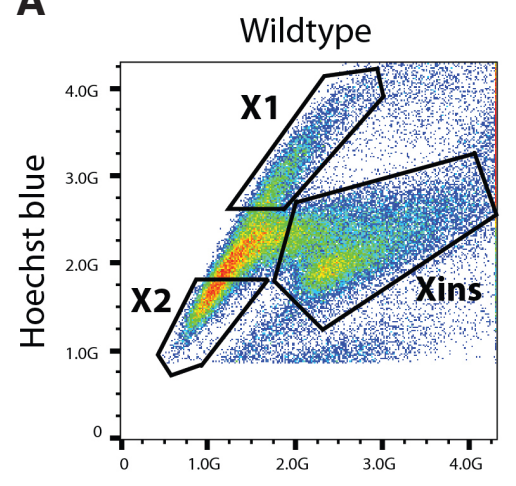

B

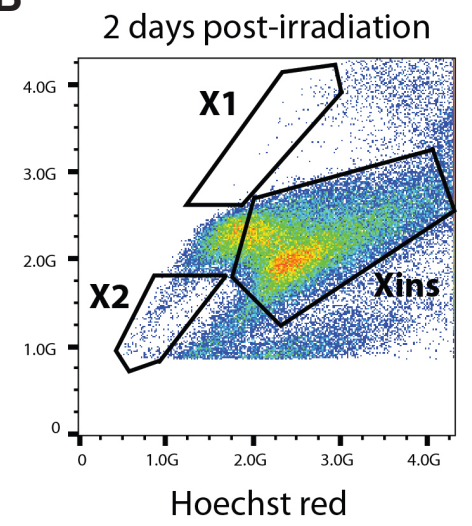

C

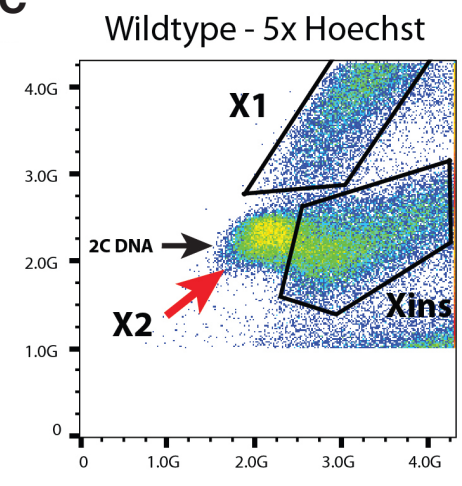

Fig. 1. Fluorescence activated cell sorting (FACS) strategy for planarian neoblasts and early progeny. FACS plots of Hoechst-stained cells in (A) unirradiated and (B) lethally irradiated animals using a previously published staining protocol (Pearson and Alvarado, 2010). (C) Increasing the Hoechst concentration 5-fold (to $125 \mathrm{\mu g} / \mathrm{ml}$ ) results in a shift of the X2 gate into the $2 \mathrm{C}$ DNA content region of the plot, demonstrating that these cells do contain 2C DNA content and normally register as Hoechst-low due to efflux of the Hoechst dye. 
and dissecting the cellular potential of any neoblast class during regeneration remains a challenge.

The detection of a wide range of tissue-specific genes within piwi-1+ neoblasts by double fluorescent ISH (dFISH) established that lineage specification is likely to occur at the neoblast level. piwi-1+lineage-marker ${ }^{+}$cells have now been identified for many different tissues. For example, sp6-9, a transcription factor required for optic cup regeneration, was observed to be expressed in a trail of eye precursor cells originating in piwi-1+ neoblasts (Lapan and Reddien, 2011; Lapan and Reddien, 2012). Likewise, the expression of transcription factors required for the regeneration of various neural subtypes has been observed in piwi-1+ neoblasts (e.g. the pitx piwi-1+ $^{+}$precursors of serotonergic neurons) (Cowles et al., 2013; Currie and Pearson, 2013). More recently, a large-scale ISH study by Scimone et al., demonstrated the non-overlapping expression of a wide variety of tissue-specific markers within piwi-1+ neoblasts isolated from the X1 FACS gate (Scimone et al., 2014). These and other such studies led to the hypothesis that there may be broad populations of lineage-committed neoblasts present in adult animals. Conversely, ideas regarding the concept of neoblast "types" versus neoblast "states" have also been articulated. These ideas postulate that, rather than maintaining lineage-committed neoblast populations, the specification of any given neoblast is stochastic and may change with the changing tissue needs of the animal (Adler and Alvarado, 2015).

The development of single-cell transcriptomics has facilitated the search for broad neoblast heterogeneity; however, further studies will likely require the establishment of in vivo lineage tracing technologies in order to rule out the stochastic model of neoblast specification. Nevertheless, putative epithelial, gut, and neural neoblast populations have already been described by a variety of methods (see below), and we will refer to them here as "lineageprimed neoblast classes". While much functional characterization remains to be performed, recent in silico evidence suggests that lineage-primed neoblast populations likely arise from a pluripotent neoblast class, termed the sigma neoblasts (Molinaro and Pearson, 2016; van Wolfswinkel et al., 2014). Unfortunately, genes originally thought to be specific to the sigma neoblast class have more recently also been detected in lineage-primed neoblast classes by single cell RNA-sequencing (scRNAseq) (Wurtzel et al., 2015). Because cell-lineage relationships are primarily assayed by RNAinterference (RNAi) knockdown of cell type-specific transcripts, it remains difficult to demonstrate conclusively that sigma neoblasts reside upstream of the lineage-primed classes. Indeed, it is possible that sigma neoblasts represent a heterogeneous mix of currently unresolved neoblast classes that may even include cNeoblasts.

\section{Epithelial-primed zeta neoblasts}

The epithelial lineage from cNeoblast $\rightarrow z f p-1^{+}$(zeta neoblast) $\rightarrow$ prog $-1^{+}$(early progenitor) $\rightarrow A G A T-1^{+}$(late progenitor) $\rightarrow$ mature epithelial cells is perhaps the best-understood planarian lineage, passing through multiple progenitor cell states over the course of 7 days of differentiation (Eisenhoffer et al., 2008; Pearson and Alvarado, 2010; Tu et al., 2015; van Wolfswinkel et al., 2014; Wurtzel et al., 2015; et al., 2017; Zhu et al., 2015). Prior to the discovery of zeta neoblasts, the work of Eisenhoffer et al., led to the discovery of the X2-enriched prog-1 and AGAT-1 neoblast progeny markers. These authors first proposed a lineage relationship from prog-1+ early neoblast progeny to $A G A T-1^{+}$late progeny based on irradiation depletion and BrdU incorporation kinetics (Eisenhoffer et al., 2008). Soon after, RNAi knockdown of another X2-enriched gene, p53, resulted in dysregulated neoblast proliferation followed by successive loss of the prog- $1^{+}$and $A G A T-1^{+}$progenitors, further supporting this lineage progression (Pearson and Alvarado, 2010). Finally, using single-cell qPCR of 96 candidate genes, van Wolfswinkel et al., identified zfp-1 as a specific marker of a subset of neoblasts, which they named the zeta neoblasts (van Wolfswinkel et al., 2014). RNAi knockdown of $z f p-1$ resulted in animal lethality caused by a failure in epithelial maintenance, and epithelial regeneration did not occur; although, remarkably, a regeneration blastema containing all other tissues still formed. Molecular analyses revealed the consecutive loss of prog-1 and AGAT-1 in zfp-1(RNAi) worms, thereby supporting the model that prog- $1^{+}$cells and $A G A T-1^{+}$cells serve respectively as early and late progenitors of the epithelial lineage (although it remains technically possible that $p r o g-1^{+}$cells may progress down other differentiation paths, given difficulties in specifically removing prog $-1^{+}$cells and the lack of a prog-1(RNAi) phenotype).

Whether zeta neoblasts are bona fide, self-renewing stem cells is unclear. Although zeta neoblasts were initially isolated from the X1 FACS gate and take up BrdU following a short 4-hour chase period, it is possible that they immediately exit the cell cycle following mitosis to begin the terminal differentiation program toward epidermis. To address the self-renewal potential of zeta neoblasts, van Wolfswinkel et al., turned to the X2 FACS gate. While often regarded as the "progeny" gate, the $\mathrm{X} 2$ gate is known to contain piwi-1+ cells, which are assumed to be G1 neoblasts (Labbé et al., 2012; Molinaro and Pearson, 2016; Zhu et al., 2015). Indeed, van Wolfswinkel et al., were successful in identifying zeta expression profiles in X2 cells. In this case, co-expression of post-mitotic epithelial lineage markers, such as prog-1, in these cells led the authors to conclude that X2 zeta neoblasts had likely exited the cell cycle, suggesting that zeta is a transient epithelial progenitor population incapable of self-renewal (van Wolfswinkel et al., 2014).

The epidermis is a heterogeneous tissue, raising the question of how zeta neoblasts become specified toward specific, spatial epithelial fates. This is a difficult question to answer given current technical limitations, but a recent scRNAseq study has uncovered a role for positional information in epithelial fate determination. In this study, single cells from the epithelial lineage were collected from dorsal, ventral and lateral regions in the animal. Differential expression analyses identified several transcripts with spatially restricted expression domains. For example, expression of PRDM1-1 within zeta neoblasts was restricted to the dorsal side of the animal, and PRDM1-1 expression in maturing cells is also restricted to the dorsal epithelium. Likewise, expression of kal1 was found in zeta neoblasts and mature epithelial cells only on the ventral side. Interestingly, expression of dorsal (PRDM1-1) and ventral (kal1) markers within zeta neoblasts was mutually exclusive (Wurtzel et al., 2017). These findings indicate that spatial cues are sensed at the earliest stage of epithelial commitment.

How positional signals are relayed back to zeta neoblasts remains to be fully elucidated, but BMP signaling has been demonstrated to play a role. Loss of BMP signaling by RNAi knockdown of bmp4 results in animal-wide ventralization. Upon bmp4 knockdown, the normally ventral $\mathrm{kal} 1^{+}$cells were observed dorsally. Intriguingly, dorsal kal1 expression was dependent on the presence of zeta neoblasts, as animals exposed to lethal irradiation prior to 
bmp4(RNAi) did not exhibit mislocalized kal1 expression (Wurtzel et al., 2017). This result suggests that at least some terminal fate decisions are made at the zeta neoblast level (Fig 2).

\section{Neural-primed nu neoblasts}

The fact that planarians can regenerate a fully functional brain in only 7-10 days following decapitation, coupled with constant homeostatic neural turnover, led to the hypothesis that neural lineage-committed neoblasts exist in the animal (Ross et al., 2017). While the presence of neoblast descendants (piwi-1- $\mathrm{PIWI}-1^{+}$) expressing neural markers have been described for multiple neural fates (Cowles et al., 2013; Currie and Pearson, 2013; März et al., 2013), more upstream, multipotent neural neoblasts were more difficult to identify in vivo. Using scRNAseq of $\mathrm{X} 1$ cells from the planarian head, our group recently described a putative neural neoblast population based on detection of a neural gene expression signature (Molinaro and Pearson, 2016). These cells, which we named nu neoblasts, expressed the neoblast marker piwi-2 as well as several known neural markers such as $p c-2$ and chat, and were surprisingly piwi-1 $1^{\mathrm{l}}$. Similar to zeta neoblasts, the selfrenewal potential of nu neoblasts is currently unknown. In BrdU pulse-chase experiments, nu neoblasts incorporate BrdU within 4 hours of administration. This, coupled with the fact that these cells were isolated from the X1 FACS gate, indicates that nu neoblasts are present during the S/G2/M phases of the cell cycle. Whether nu neoblasts subsequently exit the cell cycle to terminally differentiate or enter the G1 phase to begin a new cycle is unknown, as is what happens to neurogenesis if nu neoblasts are ablated.

Differential expression analysis comparing nu neoblasts with the remaining $X_{1}$ cells sequenced in our study demonstrated a clear enrichment of neural genes and identified several new candidate markers of this class. These markers appear to also be expressed in the brain proper by ISH and some of them are predicted to be transcription factors, perhaps suggesting a role in terminal fate selection. Functional characterization of nu neoblasts by RNAi knockdown of nu-enriched genes is a necessary next step for understanding the process of neural lineage progression in planarians. Focusing on the incorporation of new neurons into the brain in intact worms (via BrdU pulse-chase experiments) and during brain regeneration under RNAi conditions will provide important insights about the roles of nu neoblast-enriched genes in specifying neural fates.

In a separate study, our group also demonstrated a role for hedgehog signaling, in conjunction with the transcription factors $n k x 2.1$ and $a r x$, in the specification of ventral-medial neurons in the brain (Currie et al., 2016). dFISH experiments demonstrated expression of the hedgehog $(h h)$ ligand in the ventral-medial region of the brain and expression of its receptor patched-1 (ptc-1) in neighbouring neoblasts. RNAi of $h h$ resulted in a significant decrease in the production of several ventral-medial neural populations. The differentiation of planarian glia was recently reported to depend on hedgehog signaling as well. Planarian glia marked by expression of if-1 and cali are normally located within the neuropil (Wang et al., 2016). Following knockdown of ptc-1 (i.e. increasing hedgehog signaling), a dramatic increase in if- 1 $^{+} /$cali+ cells was observed outside of the neuropil. Interestingly, ptc-1 expression was not detected in nu neoblasts by scRNAseq (Molinaro and Pearson, 2016). These observations raise interesting questions regarding the specification of different neuronal fates: Do other neural lineage-primed neoblast classes exist that give rise to ventral-medial brain fates? Does a dedicated neoblast class serve to maintain glial populations? Are nu neoblasts the source of new neurons primarily in non-ventral-medial brain regions? While it remains difficult to answer these questions conclusively without tracing cells in vivo, additional scRNAseq of neoblasts from various regions surrounding the brain may provide insights on the existence of distinct neural stem cell populations, analogous to the study of spatial heterogeneity in zeta neoblasts described above. Interestingly, in a recent study that demonstrated a role for tcf- 1 in the specification of dorsal-lateral neural subtypes, several nu neoblast-markers were found to be co-expressed in tcf- $1^{\text {hi }}$ cells, perhaps suggesting a link between nu neoblasts and dorsal-lateral brain fates (Fig 3) (Brown et al., 2018).

\section{Gut-primed gamma neoblasts}

Originally identified as a subclass of the sigma class, the gamma neoblasts are predicted to be intestinal progenitors due to their expression of several known gut markers (Barberán et al., 2016; Flores et al., 2016; Forsthoefel et al., 2012; González-Sastre et al., 2017; Wagner et al., 2011; van Wolfswinkel et al., 2014). The differentiation potential of gamma neoblasts has not been directly assessed, but expression of the conserved, endodermal transcription factors $h n f 4$, gata456a, and $n k x 2.2$ in these cells is highly suggestive of intestinal fate commitment. All three factors are robustly expressed in the gut by ISH (Forsthoefel et al., 2012; Wagner et al., 2011). Knockdown of either nkx2.2 or gata456a by RNAi results in hindered gut regeneration, however, in the case of $n k x 2.2$ the gut regeneration phenotype is likely secondary to a neoblast proliferation defect (Forsthoefel et al., 2012; GonzálezSastre et al., 2017). Like zeta and nu neoblasts, their X1 origin confirms that gamma neoblasts pass through the S/G2/M phases of

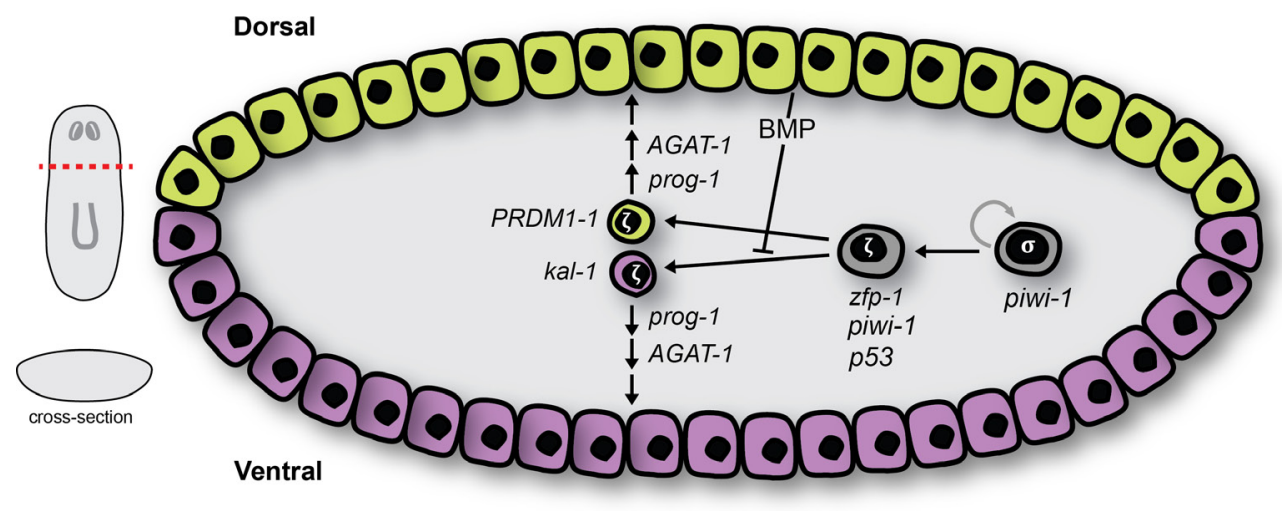

Fig. 2. Proposed zeta neoblast model. Cycling sigma $(\sigma)$ neoblasts give rise to zfp-1+ zeta (乡) neoblasts, which respond to BMP signaling and other positional cues to become specified toward dorsal or ventral epidermis. Yellow, dorsal epidermal fate; purple, ventral epidermal fate. 


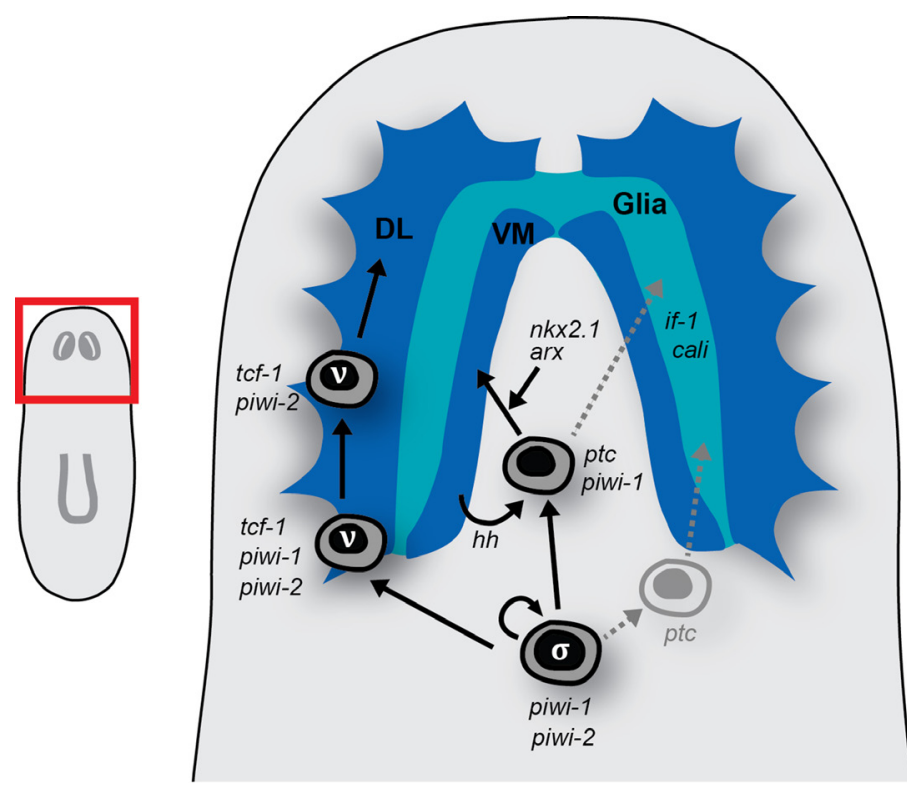

Fig. 3. Proposed nu neoblast model. Because nu $(v)$ neoblasts do not express ptc, and nu neoblast markers are expressed in tcf- $1+$ cells by scRNAseq, we propose that nu neoblasts may represent a dorsal-lateral neural progenitor. Thus, cycling sigma ( $\sigma$ ) neoblasts can give rise to dorsal-lateral nu neoblasts, which may give rise specifically to dorsal-lateral neurons, or to ptc $^{+}$ventral-medial neural neoblasts that respond to Hh signaling from the brain to give rise to ventral-medial neurons. It is currently unclear whether these ptc $^{+}$neoblasts also produce glia, or if a separate glial progenitor exists in the planarian. DL, dorsal-lateral brain; VM, ventral-medial brain.

the cell cycle, however, previous work on $h n f 4$ and $n k x 2.2$ indicate that these cells may not be self-renewing. While dFISH experiments have demonstrated mesenchymal expression of $h n f 4$ and $n k x 2.2$ in putative gamma neoblasts neighbouring the gut, these cells persist for several days following lethal irradiation, suggesting that they are downstream of cycling neoblasts (Forsthoefel et al., 2012). Conversely, irradiation does cause a loss of gata456a piwi-1+ $^{+}$cells within 24 hours, and $n k x 2$.2and $h n f 4$ expression levels were reduced following gata456a knockdown, suggesting that gata456a ${ }^{+}$piwi-1+ gamma neoblasts may represent the earliest stage of gut commitment (González-Sastre et al., 2017). In addition, RNAi knockdown of the gut-enriched EGF receptor egfr-1 resulted in an accumulation of putative gamma neoblasts around the gut and a decrease in the number of terminally differentiated intestinal cells (Barberán et al., 2016). Interestingly, egfr-1 expression was also detected in PIWI-1+ cells next to the gut (Barberán et al., 2016). This finding implicates a role for EGF signaling in the terminal differentiation of planarian gut cells and supports a role for gamma neoblasts in gut maintenance and regeneration (Fig 4).

\section{cNeoblasts: balancing adult pluripotency with controlled regeneration}

The presence of pluripotent stem cells in an adult organism is peculiar, but irrefutable evidence demonstrates this to be the case in planarians. Transplantation of a single neoblast into a lethally irradiated host, in which homeostatic turnover and regeneration cannot occur due to the lack of proliferating neoblasts, led to the clonal expansion of the donor neoblast and differentiation of its progeny along lineages from all three germ layers (Wagner et al., 2011). Further, transplantation of an asexually-derived cNeoblast into a lethally irradiated sexual host resulted in the complete replacement of host tissues, thereby switching the host genotype to that of the donor cNeoblast (Wagner et al., 2011). Thus, cNeoblasts must reside at the top of the planarian stem cell hierarchy.

At present, cNeoblasts have only been identified retrospectively by the observation of clonal expansion following sub-lethal irradiation experiments or in single cell transplants, and molecular markers have not been identified. Thus, many questions remain to be answered regarding this unusual adult stem cell population. Are cNeoblasts a maintained population (are they sigma neoblasts?) or do they arise stochastically via the de-differentiation of another neoblast class only when required (e.g. following tissue loss)? What regulatory mechanisms are in place to prevent aberrant proliferation and the development of tumors? How can we learn more about cNeoblasts without molecular markers?

\section{cNeoblasts as a discrete cell type or cell state?}

The isolation of individual cNeoblasts for transplantation experiments was performed by FACS, however, the cytotoxic effects of Hoechst meant that cells could not be sorted using the classic Hoechst-staining strategy. Instead, cNeoblasts were collected from the "X1(FS)" FACS gate, which contains cells that display forward scatter and side scatter properties similar to X1 neoblasts (Wagner et al., 2011). Due to the crudeness of this sorting strategy, the cells collected were even heterogeneous in morphology, and so the frequency of cNeoblasts is difficult to decipher. Nevertheless, because cNeoblasts were initially obtained from intact animals, it is likely that they represent a homeostatically-maintained neoblast population.

Given the abundance of multipotent neoblasts in adult planarians, the presence of actively cycling pluripotent stem cells seems to present a needless risk for the occurrence of tumorigenic mutations and subsequent teratoma formation, and such cells are not known to be common in adult organisms of other metazoans. Why, then, would

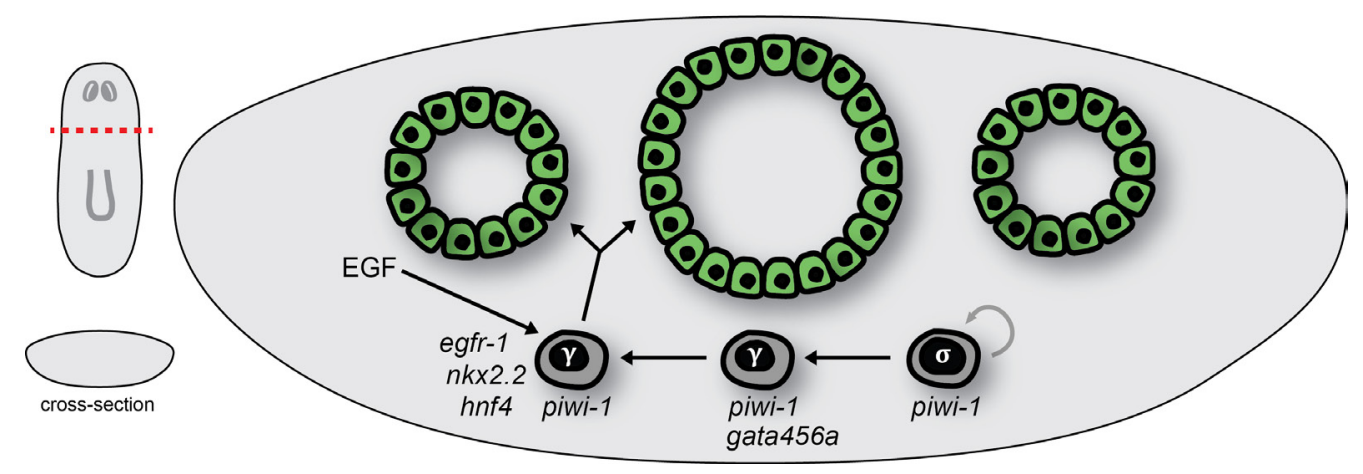

Fig. 4. Proposed gamma neoblast model. Cycling sigma ( $\sigma$ ) neoblasts give rise to gata456a+ gamma $(\gamma)$ neoblasts adjacent to the gut, which turn on expression of gut markers $\mathrm{nk} \times 2.2$ and hnf4 and begin to terminally differentiate toward intestinal fates in response to EGF signaling. Green, gut cells. 
the planarian contain such cells? The unprecedented regenerative ability of planarians perhaps justifies the necessity of cNeoblasts. If lineage-primed neoblasts are analogous to the tissue resident multipotent stem cells of vertebrates, then these neoblasts would be expected to take care of homeostatic tissue needs and minor wounds but could conceivably require back-up when regenerating entire tissues de novo. In this context, cNeoblasts would serve to replenish the lineage-primed neoblast classes during regeneration. A role for perpetually cycling cNeoblasts during homeostasis, on the other hand, is more difficult to rationalize; however, there is currently no experimental evidence to discount the possible co-existence of quiescent and actively cycling cNeoblasts.

\section{cNeoblasts as a quiescent population?}

This leads to the hypothesis that cNeoblasts may be maintained as a reserved, or quiescent, stem cell population. The non-existence of quiescent neoblasts in planarians is largely accepted, but an argument can be made that this conclusion has not been sufficiently tested. To date, the only experiments to directly ask whether slow-cycling neoblasts exist in planarians consisted of continuous BrdU administration and analysis of the fraction of labeled mitotic neoblasts (Newmark and Alvarado, 2000). With the hypothesis that slow-cycling neoblasts would not take up the BrdU label, the authors subjected worms to several BrdU injections per day for 3 days and observed that $\sim 99 \%$ of neoblasts were labeled by this strategy. However, only cell morphology of dissociated animals was used to determine whether a cell was a neoblast (i.e. no molecular markers). This result indicated that all neoblasts pass through $\mathrm{S}$ phase of the cell cycle at least once every 3 days. In addition, the authors found that by 12 hours post-BrdU administration $\sim 96 \%$ of mitotic cells (marked by $\mathrm{H} 3 \mathrm{P}$ ) were also $\mathrm{BrdU}^{+}$, indicating that all G2/M-labeled neoblasts had recently passed through S phase of the cell cycle. From this the authors reasoned that there was unlikely to be a G2-arrested population of neoblasts. What was not considered in these experiments, however, was the effect of continuous injections and repeated wound healing on neoblasts. It is plausible that this repeated wounding could provide a sufficient injury signal to activate a reserved neoblast population (Wenemoser and Reddien, 2010). The repeated incorporation of BrdU into actively cycling neoblasts would also likely result in highly cytotoxic conditions, which could conceivably lead to a depletion in the active neoblast pool by cell death and subsequent quiescent neoblast activation. To more directly test for the presence of slow-cycling, quiescent neoblasts, a pulse-chase-pulse assay using two different thymidine analogs (such as BrdU and EdU) would be more appropriate. The first pulse would be used to assay long-term label retention, while incorporation of the second pulse would confirm that these label-retaining cells are still capable of proliferation. By allowing a long chase period (perhaps several weeks) between pulses, any double-positive cells present following the second pulse must not have cycled during the chase and, therefore, would represent a quiescent population.

\section{Support for the quiescent cNeoblast hypothesis}

Interestingly, there are several examples in the planarian literature that, if viewed under the hypothesis that cNeoblasts can maintain a quiescent state, would lend support to this idea. Here we focus on two specific examples: EGF and p53 signaling.

\section{EGF signaling promotes proliferation}

The role of EGF signaling in promoting quiescent stem cell proliferation is well established in other systems. For example, quiescent neural stem cells (qNSC) in the mouse subventricular zone do not normally express the EGF receptor (EGFR) (Daynac et al., 2013). These cells are much more resistant to gammaradiation than their actively proliferating counterparts. Following irradiation-induced depletion of the active NSC pool, qNSCs have been shown to exit dormancy and acquire EGFR expression, consistent with a role for EGF signaling in promoting qNSC activation and proliferation (Daynac et al., 2013). In a similar manner, EGFR inhibition in highly proliferative Lgr5+ adult intestinal stem cells negated their ability to form intestinal organoids in culture. Molecular analysis revealed that, in contrast to wildtype Lgr5+ cells, those treated with the EGFR inhibitor lacked the general cell cycle marker Ki67 and did not incorporate EdU, indicating an induction of quiescence (Basak et al., 2017). Thus, EGF signaling is likely involved in quiescent stem cell activation.

In planarians, sub-lethal irradiation results in the loss of most neoblasts. Rare surviving neoblasts then proliferate and eventually repopulate the entire neoblast compartment, restoring homeostatic cell turnover and regenerative ability (Wagner et al., 2011; Wagner et al., 2012). Using a repopulation assay, Lei et al., recently demonstrated a role for EGF signaling in neoblast repopulation. Following sub-lethal irradiation, egfr-3(RNAi) animals were unable to repopulate the neoblast compartment (Lei et al., 2016). Importantly, homeostatic defects were not observed following egfr-3 knockdown. This suggests that the rare surviving neoblasts require EGF signaling to proliferate and act primarily during regeneration.

Several lines of evidence suggest that these rare surviving neoblasts may be quiescent cNeoblasts. First, the ability to resist higher doses of gamma-radiation is a common feature of quiescent stem cells. Second, surviving neoblasts clonally expand to repopulate the entire neoblast compartment and restore normal tissue production, indicating that the surviving neoblasts are pluripotent.

In this way, the egfr-3 RNAi phenotype could be interpreted as a defect in quiescent neoblast activation. In planarians, the regenerative response is characterized by two peaks in mitosis, the first occurring at 6 hours post-injury (hpi) and the second occurring around 48 hpi (Wenemoser and Reddien, 2010). Analysis of the mitotic response during regeneration in egfr-3(RNAi) animals revealed a slight decrease in the magnitude of the first peak and almost a complete lack of a second peak (Lei et al., 2016). The first mitotic peak, which also occurs following minor wounding and feeding (Wenemoser and Reddien, 2010), is likely the responsibility of the active neoblast compartment, possibly including the lineage-primed neoblast classes. It is conceivable that following tissue loss this first mitotic peak largely exhausts the active neoblast compartment. For successful regeneration to occur, neoblasts of all lineages must then be replenished by a reserved pluripotent neoblast population. If reserved neoblasts cannot receive activating signals, the mitotic response would be mitigated, as observed following egfr-3 knockdown (Fig 5A). This is consistent with the idea that cNeoblasts are reserved for replenishing the active neoblast compartment as it becomes depleted due to the demanding tissue requirements of regeneration. Importantly, in the case of egfr-3knockdown, the repopulation and regeneration defects were due to a decrease in proliferation, not 
an increase in cell death (Lei et al., 2016). This supports the notion that irradiation-resistant pluripotent cNeoblasts require EGF signaling to allow proliferation.

\section{p53 maintains quiescence}

p53, primarily known for its tumor suppressor activity, is an important negative regulator of proliferation (Rivlin et al., 2011). A role for p53 in maintaining quiescence in hematopoietic stem cells (HSC) has been described. p53 is highly expressed in HSCs, which, as a population, remain quiescent (Fleming et al., 1993; Foudi et al., 2009; Trumpp et al., 2010; Wilson et al., 2008; Wilson and Trumpp, 2006). While microenvironmental factors arising from the bone marrow niche likely contribute to the maintenance of HSC quiescence (Wilson and Trumpp, 2006), p53 expression has also been shown to play a role (Liu et al., 2009). In p53-/- mice, the proportion of HSCs in GO is significantly reduced while the number of cycling HSCs is increased, indicating premature activation of these cells (Liu et al., 2009). Similar findings from human fibroblasts, in which abrogation of p53 function results in activation of the normally quiescent population, further support a role for p53 in maintaining quiescence (Itahana et al., 2002).

In intact planarians, knockdown of $p 53$ by RNAi results in animal-wide neoblast hyperproliferation followed by eventual neoblast exhaustion (Pearson and Alvarado, 2010). Intriguingly, this hyperproliferation was not observed during regeneration. The original interpretation of this result was that the level of proliferation achieved during regeneration is the highest possible in planarians. An alternative interpretation revolves around p53's role in maintaining quiescence. Let us hypothesize that, in intact animals, pluripotent cNeoblasts are not required to maintain tissue homeostasis and are maintained in a quiescent state by p53. Under this hypothesis, upon p53 knockdown, these cells would become prematurely activated, causing the observed hyperproliferation and eventually leading to neoblast exhaustion. During regeneration, reserved neoblasts would normally be activated to replenish the active neoblast compartment, and so loss of quiescence at this stage should not influence proliferation levels compared to controls. Thus, the observed p53 phenotype is consistent with the expected effect of premature activation and differentiation of quiescent cNeoblasts (Fig. 5A). Interestingly, p53expression was found to be enriched in the X2 FACS gate, where it was detected in approximately $14 \%$ of cells (Pearson and Alvarado, 2010). This is notable considering that the X2 gate resembles a Hoechst side population, which have classically been found to be highly enriched for stem cells in many tissues, including multipotent quiescent stem cells (Alvi et al., 2003; Bhatt et al., 2003; Bhattacharya et al., 2003; Goodell et al., 1996; Wulf et al., 2003).

An interesting caveat of the p53 phenotype is revealed at a low RNAi dose, which results in the formation of teratoma-like outgrowths (Pearson and Alvarado, 2010). It is plausible that lowering the RNAi dose limits the phenotypic effect to a small number of cells, thereby resulting in only localized hyperproliferation. These outgrowths were found to contain all major tissue types, suggesting that an aberrantly proliferating cNeoblast may be the founding cell.
In a recent study by Cheng et al., a transcriptional hierarchy controlling epithelial lineage commitment and progression was described, at the top of which sits p53. This was not surprising, as p53 had previously been shown to be co-expressed with and affect other epithelial lineage markers (prog-1 and AGAT-1), and was detected in $z f p-1^{+}$zeta neoblasts by single cell qPCR and scRNAseq (Pearson and Alvarado, 2010; van Wolfswinkel et al., 2014; Wurtzel et al., 2017). What was interesting, however, was the discovery by Cheng et al., of $p 53^{+}$piwi- $1^{+}$cells located adjacent to the gut. These cells were found to express various combinations of zeta, gamma and sigma neoblast markers, leading the authors to speculate that these may be pluripotent neoblasts. While work regarding the planarian stem cell niche is scant and the proliferative potential of $p 53^{+}$piwi-1+ $1^{+}$cells is yet to be assessed, it is intriguing to consider that the gut epithelium might provide niche signals important for maintaining quiescent cNeoblasts (Cheng et al., 2017).

\section{FACS strategies moving forward}

\section{Strategies for studying lineage-primed neoblast classes}

In the case of the neoblast classes discussed above, we recognize these cell groups as neoblast populations primarily because they were originally isolated from the $\mathrm{X} 1$ gate, however, it is difficult to say with certainty whether these populations truly fit the definition of stem cells. While BrdU incorporation into zeta and nu neoblasts has been demonstrated after very short chase periods, it remains entirely possible that these cell types are specified during S-phase and do not re-enter the cell cycle. Indeed, a recent study by Lai et al., suggested that sigma neoblasts may be the only cycling neoblast class based on the expression of sigma-enriched genes in what were described as "giant" endocycling neoblasts, which appeared following knockdown of the condensin I subunit Smed-NCAPG (Lai et al.,
A

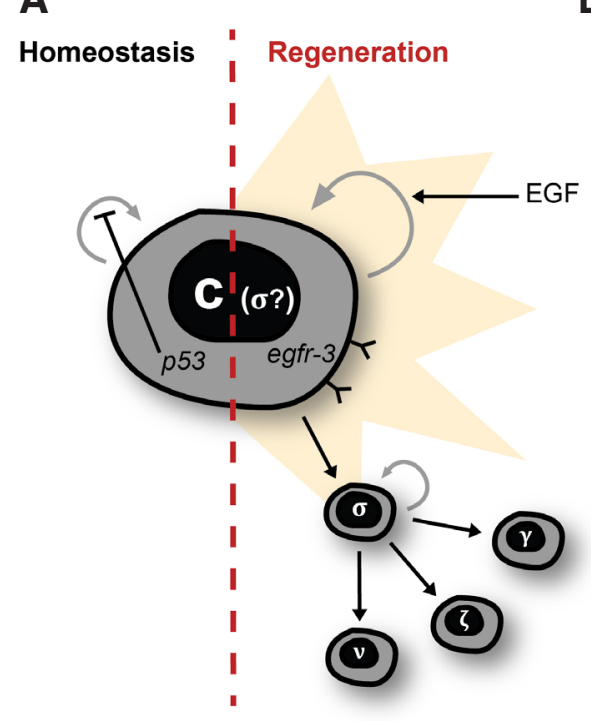

B

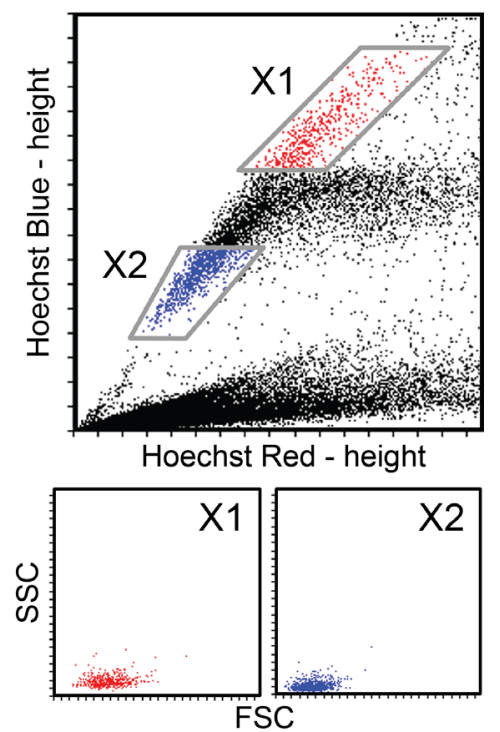

Fig. 5. Proposed cNeoblast model. (A) During homeostasis, p53 acts to inhibit the cell cycle and maintain cNeoblasts (c) in a quiescent state. During regeneration, expression of egfr-3 is increased and cNeoblasts begin to proliferate in response to EGF signaling originating from the niche. cNeoblasts reside at the top of the stem cell hierarchy in planarians and replenish all downstream neoblast populations. (B) FACS plots demonstrating the similar forward scatter (FSC) and side scatter (SSC) properties of X1 and X2 cells. 
2017). Without the ability to prospectively isolate specific neoblast classes or trace cells in vivo, it will be difficult to show definitively whether these cells are capable of self-renewal.

Closer examination of the $\mathrm{X} 2$ gate may prove to be informative for evaluating the self-renewal potential of the neoblast classes. Because G1 neoblasts likely reside in the $\mathrm{X} 2$ (or non-X1) gate, lineage-primed neoblasts capable of self-renewal should transition through this gate during the G1 phase of the cell cycle. As discussed above, transcriptional analysis of X2 zeta neoblasts has provided some insights regarding their self-renewal potential. Additional scRNAseq of the $\mathrm{X} 2$ gate may reveal zeta neoblasts that retain a general G1 neoblast expression profile. Similar analyses for the gamma and nu classes will be necessary to begin elucidating the self-renewal capacity of these cells.

As a work-around solution for the lack of prospective isolation of lineage-primed neoblasts, several studies have used qPCR to check for the presence of tissue-specific gene expression in single-cell cDNA libraries prior to sequencing (Scimone et al., 2016; Wurtzel et al., 2017). Libraries that lack expression of the gene are immediately removed from the experiment, resulting in a highly purified collection of cDNA libraries for sequencing. While effective, this approach is laborious and incompatible with newer high throughput technologies, such as Drop-seq (Macosko et al., 2015). As an alternative approach, FACS purification of fluorescently labeled cells may prove useful. Although transgenic labeling is not currently possible and cell-surface antibodies have proven hard to come by, FISH is a very well-established technique in the planarian field. Fortunately, several protocols exist describing methods for reverse-crosslinking formaldehyde fixed samples and subsequently preparing cDNA libraries for RNA-seq. One such protocol, FRISCR, is specifically designed to recover high quality RNA from single fixed cells (Thomsen et al., 2015). Granted, prospective isolation of live lineage-primed neoblasts is desirable, this FISH/FACS strategy for scRNAseq would provide an in-depth look at the transcriptional events regulating neoblast specification.

\section{Strategies for studying cNeoblasts}

Because molecular markers have not yet been identified for cNeoblasts, the techniques for neoblast class enrichment described above are not possible for this class. However, once again the X2 gate may hold some answers. As a pluripotent stem cell, cNeoblasts presumably reside at the top of the stem cell hierarchy and therefore represent bona fide stem cells. As such, cNeoblasts must also exist outside of the S/G2/M phases of the cell cycle. Although they were described to have similar physical properties as cells from the X1 gate, cells of the $\mathrm{X} 2$ gate display very similar forward and side scatter properties as X1s (Fig. 5B), therefore, it is difficult to conclude from these metrics alone whether cNeoblasts primarily reside in the $\mathrm{X} 1$ or $X 2$ gate. Considering that bona fide stem cells are often found within the Hoechst side population and that current evidence cannot rule out the hypothesis that cNeoblasts are homeostatically quiescent, the $\mathrm{X} 2$ gate seems a likely source of cNeoblasts.

\section{Future outlook}

Understanding planarian stem cell lineages is an important prerequisite for elucidating the robust regenerative mechanisms employed by these animals. While single-cell transcriptomic analysis has proven valuable, it will be necessary to establish other tools to accomplish detailed lineage analyses. Given the prior success of FACS strategies for live neoblast and progeny isolation, the production of cell surface antibodies that are compatible with live cells would be invaluable for prospectively isolating different classes of neoblasts, similar to what is done for human HSC lineage isolation. Once cells are isolated, their potency could be tested in vitro by cell culture or in vivo by transplantation into irradiated hosts. An even better approach would involve transgenically marking cells prior to transplant so all of a cell's descendants can be assayed as well. This combination of subtype isolation with lineage tracing would give definitive answers regarding the potency of a given cell, as well as whether the lineage-primed classes are competent to respond to niche signals and go back up the hierarchy to pluripotency.

\section{Acknowledgements}

This research was supported by Natural Sciences and Engineering Research Council (NSERC) grant number RGPIN-2016-06354. AMM was funded by Canadian Institutes for Health Research Banting and Best PhD fellowship GSD-152379. BJP was supported by Ontario Institute for Cancer Research (OICR) grant number IA-026.

\section{References}

ADLER C, ALVARADOA(2015). Types or States? Cellular Dynamics and Regenerative Potential. Trends Cell Biol 25: 687-696.

ALVI AJ, CLAYTON H, JOSHI C, ENVER T, ASHWORTH A, VIVANCO M d, DALE TC, SMALLEY MJ (2003). Functional and molecular characterisation of mammary side population cells. Breast Cancer Res 5: R1-8.

BARBERÁN S, FRAGUAS S, CEBRIÀ F (2016). The EGFR signaling pathway controls gut progenitor differentiation during planarian regeneration and homeostasis. Development 143: 2089-102.

BASAK O, BEUMER J, WIEBRANDS K, SENO H, VAN OUDENAARDEN A, CLEVERS H (2017). Induced Quiescence of Lgr5+ Stem Cells in Intestinal Organoids Enables Differentiation of Hormone-Producing Enteroendocrine Cells. Cell Stem Cell 20: 177-190.e4.

BHATT RI, BROWN MD, HART CA, GILMORE P, RAMANI VA, GEORGE NJ, CLARKE NW (2003). Novel method for the isolation and characterisation of the putative prostatic stem cell. Cytometry A 54: 89-99.

BHATTACHARYAS, JACKSON JD, DASAV, THORESONWB, KUSZYNSKIC, JAMES J, JOSHIS, AHMADI(2003). Direct identification and enrichment of retinal stem cells/ progenitors by Hoechst dye efflux assay. Invest Ophthalmol Vis Sci44: 2764-2773.

BROWN DDR, MOLINARO AM, PEARSON BJ (2017). The planarian TCF/LEF factor Smed-tcf1 is required for the regeneration of dorsal-lateral neuronal subtypes. Dev Biol 433: 374-383.

CEBRIÀ F (2007). Regenerating the central nervous system: how easy for planarians! Dev Genes Evol 217: 733-748.

CHENG LC, TU KC, SEIDEL CW, ROBB SMC, GUO F, SÁNCHEZ ALVARADO A (2018). Cellular, ultrastructural and molecular analyses of epidermal cell development in the planarian Schmidtea mediterranea. Dev Biol. 433: 357-373.

COWLES M, BROWN D, NISPEROS S, STANLEY B, PEARSON B, ZAYAS R (2013) Genome-wide analysis of the bHLH gene family in planarians identifies factors required for adult neurogenesis and neuronal regeneration. Development 140: 4691-4702.

CURRIE KW, MOLINARO AM, PEARSON BJ (2016). Neuronal sources of hedgehog modulate neurogenesis in the adult planarian brain. eLife 2016 Nov 19;5.

CURRIE KW, PEARSON BJ (2013). Transcription factors Ihx1/5-1 and pitx are required for the maintenance and regeneration of serotonergic neurons in planarians. Development 140: 3577-3588.

DAYNACM, CHICHEPORTICHEA, PINEDAJ, GAUTHIERL, BOUSSINF, MOUTHON $M-A$ (2013). Quiescent neural stem cells exit dormancy upon alteration of GABAAR signaling following radiation damage. Stem Cell Res 11: 516-528.

DUBOIS F (1949). Contribution a l'étude de la migration des cellules de régénération chez les planaires dulcicoles. Bull Biol France et Belg 83: 213-283. 
EISENHOFFER G, KANG H, ALVARADO A (2008). Molecular Analysis of Stem Cells and Their Descendants during Cell Turnover and Regeneration in the Planarian Schmidtea mediterranea. Cell Stem Cell 3: 327-339.

FLEMING WH, ALPERN EJ, UCHIDA N, IKUTA K, SPANGRUDE GJ, WEISSMAN IL (1993). Functional heterogeneity is associated with the cell cycle status of murine hematopoietic stem cells. J Cell Biol 122: 897-902.

FLORES NM, OVIEDO NJJ, SAGE J (2016). Essential role for the planarian intestinal GATA transcription factor in stem cells and regeneration. Dev Biol 418: 179-188.

FORSTHOEFEL DJ, JAMES NPP, ESCOBAR DJ, STARY JM, VIEIRA AP, WATERS FA, NEWMARK PA (2012). An RNAi screen reveals intestinal regulators of branching morphogenesis, differentiation, and stem cell proliferation in planarians. Dev Cell 23: 691-704.

FOUDI A, HOCHEDLINGER K, VAN BUREN D, SCHINDLER JW, JAENISCH R, CAREY V, HOCK H (2009). Analysis of histone 2B-GFP retention reveals slowly cycling hematopoietic stem cells. Nat Biotechnol 27: 84-90.

GONZÁLEZ-SASTRE A, DE SOUSA N, ADELL T, SALÓ E (2017). The pioneer factor Smed-gata456-1 is required for gut cell differentiation and maintenance in planarians. Int J Dev Biol 61: 53-63.

GOODELLMA, BROSEK, PARADIS G, CONNERAS, MULLIGANRC (1996). Isolation and functional properties of murine hematopoietic stem cells that are replicating in vivo. J Exp Med 183: 1797-1806.

HAYASHI T, ASAMI M, HIGUCHI S, SHIBATA N, AGATA K (2006). Isolation of planarian X-ray-sensitive stem cells by fluorescence-activated cell sorting. Dev Growth Differ 48: 371-380.

ITAHANA K, DIMRI GP, HARA E, ITAHANA Y, ZOU Y, DESPREZ P-YY, CAMPISI J (2002). A role for p53 in maintaining and establishing the quiescence growth arrest in human cells. J Biol Chem 277: 18206-18214.

LABBÉ R, IRIMIA M, CURRIE K, LIN A, ZHU S, BROWN D, ROSS E, VOISIN V, BADER G, BLENCOWE B, PEARSON B (2012). A Comparative Transcriptomic Analysis Reveals Conserved Features of Stem Cell Pluripotency in Planarians and Mammals. Stem Cells 30: 1734-1745.

LAI AG, KOSAKA N, ABNAVE P, SAHU S, ABOOBAKERAA (2017). The abrogation of condensin function provides independent evidence for defining the self-renewing population of pluripotent stem cells. Dev. Biol. 433: 218-226.

LAPAN S, REDDIEN P (2011). dlx and sp6-9 Control optic cup regeneration in a prototypic eye. PLoS genetics 7: e1002226.

LAPAN S, REDDIEN P (2012). Transcriptome Analysis of the Planarian Eye Identifies ovo as a Specific Regulator of Eye Regeneration. Cell Reports 2: 294-307.

LEI K, THI-KIM VU H, MOHAN RD, MCKINNEY SA, SEIDEL CW, ALEXANDER R, GOTTING K, WORKMAN JL, SÁNCHEZ ALVARADO A (2016). Egf Signaling Directs Neoblast Repopulation by Regulating Asymmetric Cell Division in Planarians. Dev Cell 38: 413-429.

LIU Y, ELF SE, MIYATA Y, SASHIDA G, LIU Y, HUANG G, DI GIANDOMENICO S, LEE JM, DEBLASIO A, MENENDEZ S, ANTIPIN J, REVA B, KOFF A, NIMER SD (2009). p53 regulates hematopoietic stem cell quiescence. Cell Stem Cell 4:37-48.

MACOSKO EZ, BASUA, SATIJAR, NEMESHJ, SHEKHARK, GOLDMAN M, TIROSH I, BIALAS AR, KAMITAKI N, MARTERSTECK EM, TROMBETTA JJ, WEITZ DA, SANES JR, SHALEK AK, REGEV A, MCCARROLL SA (2015). Highly Parallel Genome-wide Expression Profiling of Individual Cells Using Nanoliter Droplets. Cell 161: 1202-1214.

MÄRZ M, SEEBECK F, BARTSCHERER K (2013). A Pitx transcription factor controls the establishment and maintenance of the serotonergic lineage in planarians. Development 140: 4499-4509.

MOLINAROA, PEARSONB (2016). In silico lineage tracing through single cell transcriptomics identifies a neural stem cell population in planarians. Genome Biol 17: 87.

NEWMARK P, ALVARADO A (2000). Bromodeoxyuridine specifically labels the regenerative stem cells of planarians. Dev Biol 220: 142-153.

NEWMARK P, ALVARADO A (2002). Not your father's planarian: a classic model enters the era of functional genomics. Nat Rev Genet 3: 210-219.

PEARSON BJ, SÁNCHEZ ALVARADO A (2010). A planarian p53 homolog regulates proliferation and self-renewal in adult stem cell lineages. Development 137:213-221.

PELLETTIERI J, ALVARADO A (2007). Cell turnover and adult tissue homeostasis: from humans to planarians. Annu Rev Genet 41: 83-105.
RANDOLPH H (1897). Observations and experiments on regeneration in planarians. Dev Genes Evol 5: 352-372.

RANDOLPHH (1892). The regeneration of the tail in Lumbriculus. JMorphol7:317-344.

REDDIEN P, ALVARADO A (2004). Fundamentals of planarian regeneration. Annu Rev Cell Dev Bi 20: 725-757.

REDDIEN P, OVIEDO N, JENNINGS J, JENKIN J, ALVARADO A (2005). SMEDWI-2 is a PIWI-like protein that regulates planarian stem cells. Science 310: 1327-1330.

RINK J (2013). Stem cell systems and regeneration in planaria. Dev Genes Evol 223: 67-84.

RIVLIN N, BROSH R, OREN M, ROTTER V (2011). Mutations in the p53 Tumor Suppressor Gene: Important Milestones at the Various Steps of Tumorigenesis. Genes \& Cancer 2: 466-474.

ROSS KG, CURRIE KW, PEARSON BJ, ZAYAS RM (2017). Nervous system development and regeneration in freshwater planarians. Wiley Interdiscip Rev Dev Biol. 2017 May; 6(3).

SCIMONE ML, COTE LE, ROGERS T, REDDIEN P (2016). Two FGFRL-Wnt circuits organize the planarian anteroposterior axis. eLife 2016 Apr 11;5

SCIMONE ML, KRAVARIK KM, LAPAN SW, REDDIEN PW (2014). Neoblast specialization in regeneration of the planarian Schmidtea mediterranea. Stem Cell Rep 3: 339-352.

SHERLEY JL (2002). Asymmetric Cell Kinetics Genes: The Key to Expansion of Adult Stem Cells in Culture. Sci World J 2: 1906-1921.

THOMSEN E, MICH J, YAO Z, HODGE R, DOYLE A, JANG S, SHEHATAS, NELSON A, SHAPOVALOVA N, LEVI B, RAMANATHAN S (2015). Fixed single-cell transcriptomic characterization of human radial glial diversity. Nat Methods 13: 87-93.

TRUMPPA, ESSERS M, WILSON A (2010). Awakening dormant haematopoietic stem cells. Nat Rev Immunol 10: 201-209.

TU K, CHENG L-C, VU H, LANGE J, MCKINNEY S, SEIDEL C, ALVARADO A (2015). Egr-5 is a post-mitotic regulator of planarian epidermal differentiation. eLife 4: e10501.

WAGNER D, WANG I, REDDIEN P (2011). Clonogenic Neoblasts Are Pluripotent Adult Stem Cells That Underlie Planarian Regeneration. Science 332: 811-816.

WAGNER DE, HO JJ, REDDIEN P (2012). Genetic regulators of a pluripotent adult stem cell system in planarians identified by RNAi and clonal analysis. Cell Stem Cell 10: 299-311.

WANG IE, LAPAN SW, SCIMONE ML, CLANDININ TR, REDDIEN PW (2016). Hedgehog signaling regulates gene expression in planarian glia. eLife 2016 Sep 9;5.

WENEMOSER D, REDDIEN P (2010). Planarian regeneration involves distinct stem cell responses to wounds and tissue absence. Dev Biol 344: 979-991.

WILSON A, TRUMPP A (2006). Bone-marrow haematopoietic-stem-cell niches. Nat Rev Immunol. 6: 93-106.

WILSONA, LAURENTIE, OSERG, VANDER WATHRC, BLANCO-BOSEW, JAWORSKI M, OFFNER S, DUNANT CF, ESHKIND L, BOCKAMP E, LIÓ P, MACDONALD HR, TRUMPP A (2008). Hematopoietic stem cells reversibly switch from dormancy to self-renewal during homeostasis and repair. Cell 135: 1118-1129.

VANWOLFSWINKELJ, WAGNERD, REDDIEN P (2014). Single-Cell Analysis Reveals Functionally Distinct Classes within the Planarian Stem Cell Compartment. Cell Stem Cell 15: 326-339.

WENEMOSER D, REDDIEN P (2010). Planarian regeneration involves distinct stem cell responses to wounds and tissue absence. Dev Biol 344: 979-991.

WULF GG, LUO K-LL, JACKSON KA, BRENNER MK, GOODELL MA (2003). Cells of the hepatic side population contribute to liver regeneration and can be replenished with bone marrow stem cells. Haematologica 88: 368-378.

WURTZEL O, COTE LE, POIRIER A, SATIJA R, REGEV A, REDDIEN PW (2015). A Generic and Cell-Type-Specific Wound Response Precedes Regeneration in Planarians. Dev Cell 35: 632-645.

WURTZEL O, ODERBERG I, REDDIEN P (2017). Planarian Epidermal Stem Cells Respond to Positional Cues to Promote Cell-Type Diversity. Dev Cell40:491-504.e5.

ZHU S, HALLOWS S, CURRIE K, XU C, PEARSON B (2015). A mex3 homolog is required for differentiation during planarian stem cell lineage development. eLife 2015 Jun 26;4.

ZHU S, PEARSON B (2016). (Neo)blast from the past: new insights into planarian stem cell lineages. Curr Opin Genet Dev 40: 74-80. 


\section{Further Related Reading, published previously in the Int. J. Dev. Biol.}

Planaria: an animal model that integrates development, regeneration and pharmacology

Oné R. Pagán

Int. J. Dev. Biol. (2017) 61: 519-529

https://doi.org/10.1387/ijdb.160328op

The pioneer factor Smed-gata456-1 is required for gut cell differentiation and maintenance in planarians Alejandro González-Sastre, Nídia De Sousa, Teresa Adell and Emili Saló

Int. J. Dev. Biol. (2017) 61: 53-63

https://doi.org/10.1387/ijdb.160321es

The natural compound sanguinarine perturbs the regenerative capabilities of planarians

Linda Balestrini, Alessia Di Donfrancesco, Leonardo Rossi, Silvia Marracci, Maria E. Isolani, Anna M. Bianucci and Renata Batistoni

Int. J. Dev. Biol. (2017) 61: 43-52

https://doi.org/10.1387/ijdb.160169rb

Innate sexuality determines the mechanisms of telomere maintenance

Kenta Tasaka, Naoki Yokoyama, Hanae Nodono, Motonori Hoshi and Midori Matsumoto

Int. J. Dev. Biol. (2013) 57: 69-72

https://doi.org/10.1387/ijdb.120114mm

Regeneration of neuronal cell types in Schmidtea mediterranea: an immunohistochemical and expression study

Susanna Fraguas, Sara Barberán, Begoña Ibarra, Linda Stöger and Francesc Cebrià

Int. J. Dev. Biol. (2012) 56: 143-153

https://doi.org/10.1387/ijdb.113428sf

Stem cells and neural signalling: the case of neoblast recruitment and plasticity in low dose X-ray treated planarians

Leonardo Rossi, Paola lacopetti and Alessandra Salvetti

Int. J. Dev. Biol. (2012) 56: 135-142

https://doi.org/10.1387/ijdb.123505lr

Stem cell protection mechanisms in planarians: the role of some heat shock genes Maria-Emilia Isolani, Maria Conte, Paolo Deri and Renata Batistoni

Int. J. Dev. Biol. (2012) 56: 127-133

https://doi.org/10.1387/ijdb.113432mi

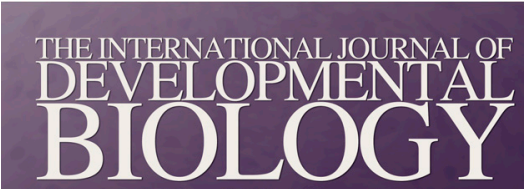

Volume 60 Nos. 10/11/12 Special Issue

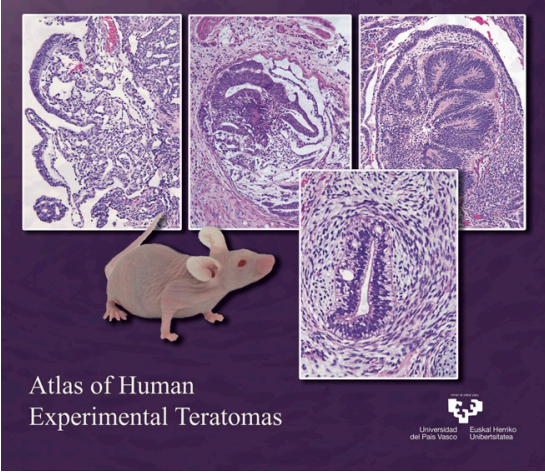

5 yr ISI Impact Factor $(2016)=2.421$

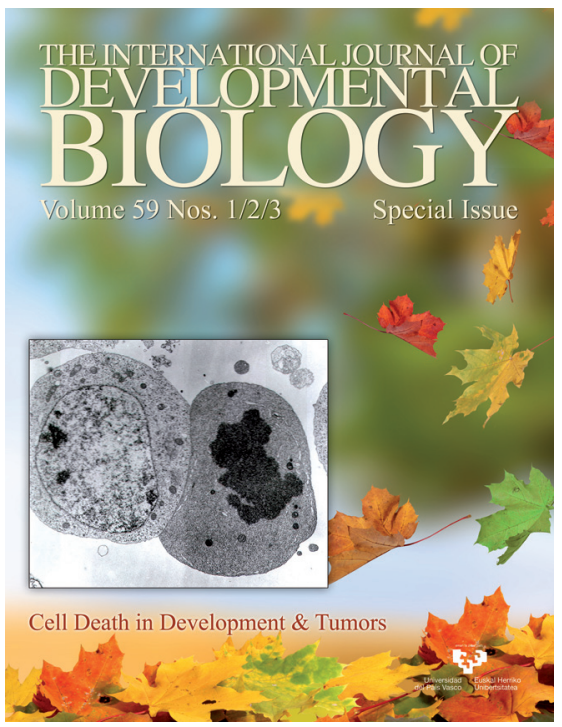

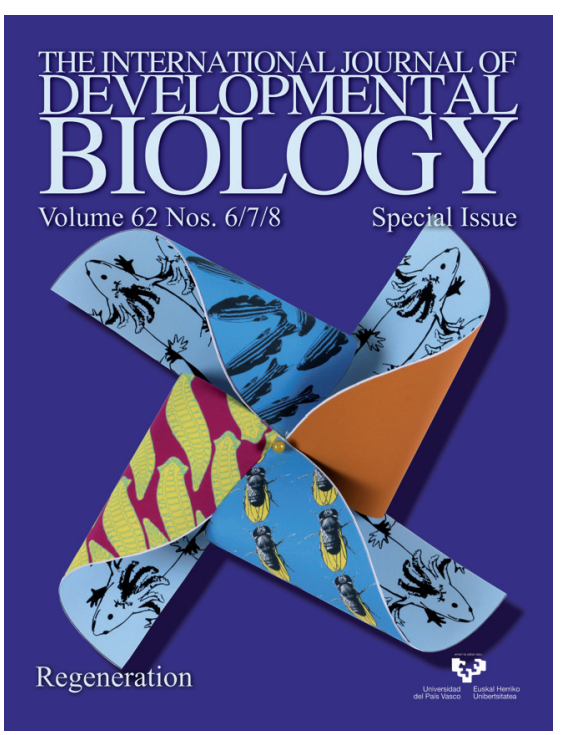

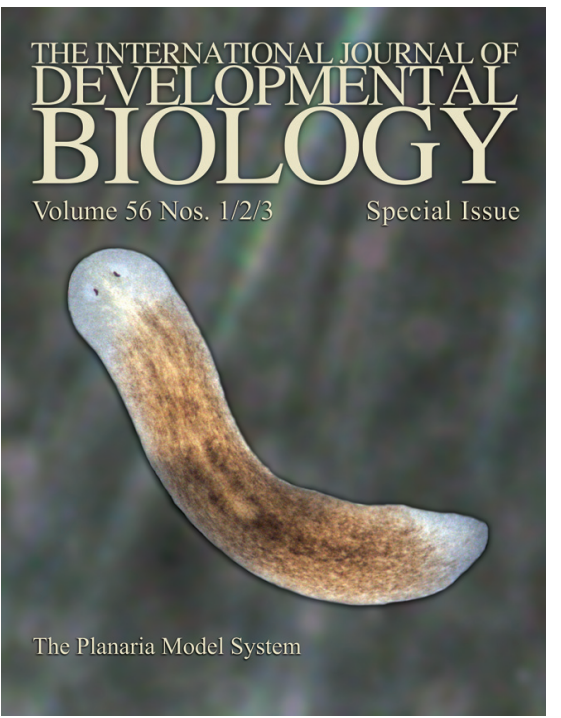

